\title{
Amygdala Neurons Differentially Encode Motivation and Reinforcement
}

\author{
Kay M. Tye ${ }^{1,2}$ and Patricia H. Janak ${ }^{1,3}$ \\ ${ }^{1}$ Ernest Gallo Clinic and Research Center, University of California, San Francisco, Emeryville, California 94608, and ${ }^{2}$ Graduate Program in Neuroscience and \\ ${ }^{3}$ Department of Neurology and Wheeler Center for the Neurobiology of Addiction, University of California, San Francisco, San Francisco, California 94143
}

Lesion studies demonstrate that the basolateral amygdala complex (BLA) is important for assigning motivational significance to sensory stimuli, but little is known about how this information is encoded. We used in vivo electrophysiology procedures to investigate how the amygdala encodes motivating and reinforcing properties of cues that induce reinstatement of reward-seeking behavior. Two groups of rats were trained to respond to a sucrose reward. The "paired" group was trained with a reward-predictive cue, whereas the "unpaired" group was trained with a randomly presented cue. Both groups underwent identical extinction and reinstatement procedures during which the reward was withheld. The proportion of neurons that were phasically cue responsive during reinstatement was significantly higher in the paired group ( 46 of 100) than in the unpaired group ( 8 of 112). Cues that induce reward-seeking behavior can do so by acting as incentives or reinforcers. Distinct populations of neurons responded to the cue in trials in which the cue acted as an incentive, triggering a motivated reward-seeking state, or as a reinforcer, supporting continued instrumental responding. The incentive motivation-encoding population of neurons ( 34 of 46 cue-responsive neurons; 74\%) extinguished in temporal agreement with a decrease in the rate of instrumental responding. The conditioned reinforcement-encoding population of neurons (12 of 46 cue-responsive neurons; $26 \%$ ) maintained their response for the duration of cue-reinforced instrumental responding. These data demonstrate that separate populations of cue-responsive neurons in the BLA encode the motivating or reinforcing properties of a cue previously associated with a reward.

Key words: relapse; cue-induced reinstatement; in vivo electrophysiology; addiction; appetitive conditioning; reward

\section{Introduction}

Understanding how environmental stimuli can acquire motivating and reinforcing properties when associated with a reward is essential for improving therapeutic interventions for psychopathologies such as obsession, depression, eating disorders, and drug addiction. In humans, reward-associated cues can increase reward-seeking behavior. Specifically, presentation of cocaineassociated stimuli to abstinent cocaine addicts produces intense drug craving (O’Brien et al., 1998), physiological arousal, including increased heart rate and skin conductance (Childress et al. 1988; Ehrman et al. 1992), and amygdala activation (Childress et al. 1999), all of which may lead to a relapse of drug-seeking behavior.

The ability of environmental cues to control reward-seeking behavior can be explored using animal models. Rats readily lever press to self-administer primary reinforcers, such as sucrose, cocaine, or alcohol, and subsequently to obtain reward-paired cues

\footnotetext{
Received Dec. 6, 2006; revised Jan. 29, 2007; accepted Feb. 26, 2007.

This work was supported by funding from the State of California for Medical Research on Alcohol and Substance Abuse through the University of California at San Francisco and a National Science Foundation Graduate Research Fellowship (K.M.T.). We would like to thank Drs. H. L. Fields, A. J. Doupe, N. Chaudhri, and L. H. Corbit for valuable discussion and W. W. Schairer for data analysis assistance.

Correspondence should be addressed to Patricia H. Janak, Ernest Gallo Clinic and Research Center, Department of Neurology, University of California, San Francisco, 5858 Horton Street, Suite 200, Emeryville, CA 94608. E-mail: pjanak@gallo.ucsf.edu.

D01:10.1523/JNEUROSC1.5281-06.2007

Copyright $\odot 2007$ Society for Neuroscience $\quad 0270-6474 / 07 / 273937-09 \$ 15.00 / 0$
}

in the absence of reward (Davis and Smith 1976; Meil and See 1997; Grimm et al. 2002; Nie and Janak, 2003). Specifically, when animals are trained to respond to a reward that is paired with a predictive cue and responding is subsequently extinguished by the omission of the cue and reward, presentation of the cue alone increases responding. This phenomenon is called cue-induced reinstatement and is an animal model for relapse to rewardseeking behavior (Epstein et al., 2006).

A single conditioned stimulus (CS) can function as both an incentive and a reinforcer for different behavioral or environmental contingencies (Everitt and Robbins, 2005). The rewardassociated cue in the cue-induced reinstatement model is typically considered to act as a conditioned reinforcer. A conditioned reinforcer is defined as a neutral stimulus that acquires reinforcing properties when associated with primary reinforcement, and conditioned reinforcement can be measured by the ability of the cue to support instrumental responding in the absence of the primary reinforcer (Arroyo et al., 1998; Everitt and Robbins, 2000, 2005). In contrast, a conditioned incentive is defined as a neutral stimulus that acquires motivating properties when associated with primary reinforcement, measured by behaviors such as conditioned approach (Berridge and Robinson, 2003).

The basolateral amygdala complex (BLA) is critically involved in the formation and expression of associations between sensory cues and rewarding or aversive stimuli (Davis, 1992; LeDoux, 1996; Gallagher, 2000; McGaugh, 2002). Amygdala neurons re- 
spond to sensory stimuli paired with positive or negative outcomes (Quirk et al., 1995; Uwano et al., 1995). BLA lesions attenuate responding to a cue associated with sexual reinforcement but do not alter sexual behavior itself (Everitt et al. 1989). Furthermore, BLA lesions do not alter cocaine self-administration but attenuate the ability of cocaine-associated cues to reinstate extinguished responses (Meil and See, 1997). This evidence suggests that BLA function is specific to reinforcing properties of the reward-associated cue and does not affect reinforcing properties of the reward itself (Balleine et al., 2003).

Because the BLA is critical for stimulus-reward learning and cue-induced reinstatement, we hypothesized that BLA neurons encode the cue-reward association that endows the cue with the power to motivate responding to a reward. To test this hypothesis, we used in vivo extracellular recording of BLA neurons during cue-induced reinstatement of reward-seeking behavior.

\section{Materials and Methods}

\section{Electrophysiological recordings}

Male Sprague Dawley rats (Harlan, Indianapolis, IN) were bilaterally implanted with electrodes in the BLA for chronic neural recording during cue-induced reinstatement of reward-seeking behavior. Rats were anesthetized with isoflurane and stereotaxically implanted bilaterally with fixed-wire electrodes with an eight-wire $2 \times 2 \times 3$ array, in which each wire was insulated and $50 \mu \mathrm{m}$ in diameter (NeuroBiological Laboratories, Denison, TX), directed at the BLA (anteriorposterior, -2.8 to -3.6 ; mediolateral, \pm 5.0 ; dorsoventral, -7.2 to -7.5 ). Rats were allowed to heal for 7-10 d, during which time they received food and water ad libitum. After healing, rats were food restricted to $90 \%$ of their ad libitum consumption for $3 \mathrm{~d}$ before performing the behavioral task. All procedures were approved by the Gallo Center Institutional Animal Care and Use Committee and were in accordance with National Institute of Health guidelines. Every attempt was made to minimize the number of animals required and to minimize their suffering.

\section{Behavioral paradigms}

Animals were divided into two groups, a "paired" group and an "unpaired" group. Animals in the paired group were trained to perform a nosepoke response that resulted in a cue presentation and sucrose delivery to an adjacent port. To demonstrate that neural responses were a result of the cue-reward association and not unconditioned sensory responses, we included an unpaired group of animals. Animals in the unpaired group were also trained to respond to sucrose delivery, but the cue was presented at random intervals, independent of other experimental events. For each $2 \mathrm{~h}$ session run on consecutive days, animals were placed into the behavioral chamber with their headstages plugged into a cable attached to a freely rotating commutator at the top of the box to enable unrestricted movement. This behavioral chamber was equipped with a nosepoke hole and adjacent reward-delivery port. A continuous house light and white noise $(60 \mathrm{db})$ signaled the onset of the behavioral program.

Training fixed-ratio 1: sessions 1-3. For both groups, animals were trained in a fixed-ratio 1 (FR1) reinforcement schedule for one session per day for $3 \mathrm{~d}$, and during the first $2 \mathrm{~d}$ of training a palatable odor cue was presented to encourage nosepoke responding. Nosepoke responses and port entries were measured by beam breaks. For the paired group, nosepoke responses were reinforced on an FR1 schedule with a subsequent (onset $50 \mathrm{~ms}$ after nosepoke) compound light-tone cue (LT-cue), $3 \mathrm{KHz}$ tone at $80 \mathrm{db}$, and illumination of two additional lights lasting $5 \mathrm{~s}$. Two seconds after the nosepoke, $0.1 \mathrm{ml}$ of a $15 \%$ sucrose solution was delivered to the port over the course of $3 \mathrm{~s}$. For the unpaired group, nosepoke responses were also reinforced on an FR1 schedule, with the same $2 \mathrm{~s}$ delay until onset of the $3 \mathrm{~s}$ sucrose solution delivery. However, the unpaired group was presented with the LT-cue at responseindependent random variable intervals. The frequency of the LT-cue presentations was matched to experimental animals so that both groups received the same approximate average number of LT-cue presentations. For both groups, animals that did not meet the learning criterion (75 nosepokes by the third training session) did not move on to the next training sessions and were excluded from the study. Two rats, one from each group, were excluded from the study for failure to meet learning criterion after $3 \mathrm{~d}$ of training.

Training random ratio 2: sessions 4-6. For the fourth session, both groups of animals were switched to a random ratio 2 reinforcement schedule in which only $50 \%$ of randomly selected nosepokes were reinforced, whereas the other $50 \%$ were recorded but not reinforced. For the paired group, reinforced nosepokes were paired with the same $50 \mathrm{~ms}$ delay until onset of the $5 \mathrm{~s}$ LT-cue in combination with the $2 \mathrm{~s}$ delay until onset of the $3 \mathrm{~s}$ sucrose delivery. For the unpaired group, reinforced nosepokes were followed with a $2 \mathrm{~s}$ delay until the onset of the $3 \mathrm{~s}$ sucrose delivery, but $5 \mathrm{~s}$ LT-cues were still presented at random intervals, occurring regardless of sucrose deliveries.

Extinction: sessions 7-8. Both groups were run on the same extinction paradigm. During this paradigm, nosepoke responses were recorded but not reinforced. Both the LT-cue and sucrose deliveries were completely omitted for the duration of the session.

Reinstatement test day: session 9. Both groups were run on the same reinstatement paradigm. During this paradigm, 50\% of nosepokes were paired with the LT-cue, and the other $50 \%$ of nosepokes were not paired with any stimuli. All nosepoke responses and port entries were recorded. For both groups, nosepoke responses were not reinforced with sucrose deliveries. For the paired group, the above situation was consistent for the entire session. For the unpaired group, because the level of responding was so low (animals did not show a cue-induced reinstatement of reward-seeking behavior), after 80 min of the above situation, four noncontingent presentations of sucrose solution were passively delivered to encourage nosepoking, both to demonstrate that the animal learned the task and was able to recall the association between nosepoke responding and sucrose deliveries and to yield more trials to allow for data analysis. All programs were written in MED-PC, and behavioral data were sent to Plexon Instruments (Dallas TX) in correspondence with neural recordings.

\section{Histology}

After the completion of the last session, animals were anesthetized with isoflurane and decapitated. A $19 \mu \mathrm{A}$ current was passed through each recording electrode that had identifiable single units. The brains were fixed with $10 \%$ formalin and $3 \%$ potassium ferricyanide and submerged in $20 \%$ sucrose and $3 \%$ potassium ferricyanide overnight. Potassium ferricyanide was used to determine the location of the electrode tip. The brains were sectioned $(50 \mu \mathrm{m})$ throughout the extent of the amygdala. Alternating sections were stained with neutral red and Nissl staining, allowing the visualization of the blue electrode placement mark in relationship to the subnuclei of the amygdala. Serial sections were examined under a light microscope, and the location of each electrode tip was plotted on coronal sections taken from the rat stereotaxic atlas of Paxinos and Watson (1998).

\section{Single-unit recording and discrimination}

Neural activity was recorded with commercial hardware and software, including headstage amplifiers to programmable amplifiers, filters $(0.5$ and $5 \mathrm{KHz} ; 3 \mathrm{~dB}$ cutoffs), and multichannel spike-sorting software (Plexon Instruments). Discrimination of individual units was performed off-line using principal component analysis of waveform shape. Single units were identified by constancy of waveform shape, crosscorrelogram, autocorrelogram, and interspike interval. Action potential durations were measured from the initial inflection of the waveform to the first trough (microseconds).

\section{Analyzing neural response properties}

A total of 10 rats ( $n=5$ rats per group) successfully recovered from surgery, met learning criterion, and were histologically confirmed to have electrode tips in the BLA. These 10 rats were included in data analyses. Sorted files were processed in NeuroExplorer to extract these unit timestamps and relevant reference event markers. Neural activity was characterized via perievent raster and perievent histogram displays. NeuroExplorer-extracted timestamps were then exported to MATLAB (Natick, MA) and analyzed for statistical significance. Responses were 
deemed statistically significant if any $100 \mathrm{~ms}$ bins in the response window $(0-0.5 \mathrm{~s}$ after the nosepoke) were statistically significant relative to a baseline epoch, a $0.5 \mathrm{~s}$ period after the animal had left the port on the previous trial and before the animal had initiated movement toward the nosepoke hole $(-2$ to $-1.5 \mathrm{~s})$. Significance was tested using the nonparametric Wilcoxon signed-rank test, with a certainty of $p<0.01$. For analysis of neural responses to cues followed by port entry versus cues not followed by port entry, variables were created on NeuroExplorer. Because of animal variability in average latency from nosepoke or cue presentation to port entry, the time epoch after a nosepoke or cue presentation in which a port entry was considered to have followed the reference event was 1.5 SDs above the mean latency for each animal. Early reinstatement was deemed to be the initial portion of the reinstatement session, during which time the animal displayed postcue port checking, whereas late reinstatement referred to trials after the time point when the latency between the nosepoke and the port entry increased by a minimum threshold of 1.5 SDs in a moving 10-trial block.

\section{Burst analysis}

Sorted neural data files were imported into NeuroExplorer. Burst analysis was performed using interval specifications such that successive spikes with an interspike interval $\leq 0.10 \mathrm{~s}$ were included within a burst with a minimum of two spikes per burst. To compare the paired group to the unpaired group, a $1000 \mathrm{~s}$ interval beginning after the time of operant response initiation was selected such that both groups had similar levels of activity and cue presentations (paired, 1000-2000 s; unpaired, 5600$6600 \mathrm{~s})$.

\section{Results}

A diagram of the behavioral paradigm is shown in Figure 1. All neurons from the paired and unpaired groups included in the analysis were recorded from electrodes located in the BLA (Fig. $2 A)$. Individual neurons were isolated by waveform template (Fig. 2B) and principal component cluster analysis.

\section{Cue-reward association is necessary for cue-induced reinstatement of reward-seeking behavior}

The paired and unpaired groups demonstrated similar levels of nosepoke responding for all sessions except the reinstatement session. Here, paired animals demonstrated reinstatement of reward-seeking behavior, indicated by a significant increase in nosepoke responses relative to extinction. In contrast, unpaired animals failed to reinstate extinguished responding (Fig. $3 A$ ). To demonstrate that animals in the unpaired group were capable of reinstating extinguished nosepoke responding, after $80 \mathrm{~min}$ of the reinstatement session, four noncontingent "priming" sucrose rewards were delivered at random intervals (Fig. 3B). All unpaired animals demonstrated reinstatement of nosepoke responding after sucrose priming, indicating that they were capable of reinstating the extinguished nosepoke response that was associated with the reinforcer. In addition, the sucrose-primed reinstatement served to increase the number of trials available to compare with the paired animals in analysis.

The presence or absence of the cue guided the behavioral response pattern of paired but not unpaired animals (Fig. 3C). Specifically, within $2 \mathrm{~s}$ after the cue, paired animals were 75 times more likely to enter the port than to nosepoke again, whereas unpaired animals had a similar likelihood of port checking or nosepoking. In the $2 \mathrm{~s}$ after a nosepoke without the cue, paired animals were 3.4 times more likely to repeat a nosepoke response than to enter the port, whereas unpaired animals had a similar likelihood of nosepoke responding or port checking.

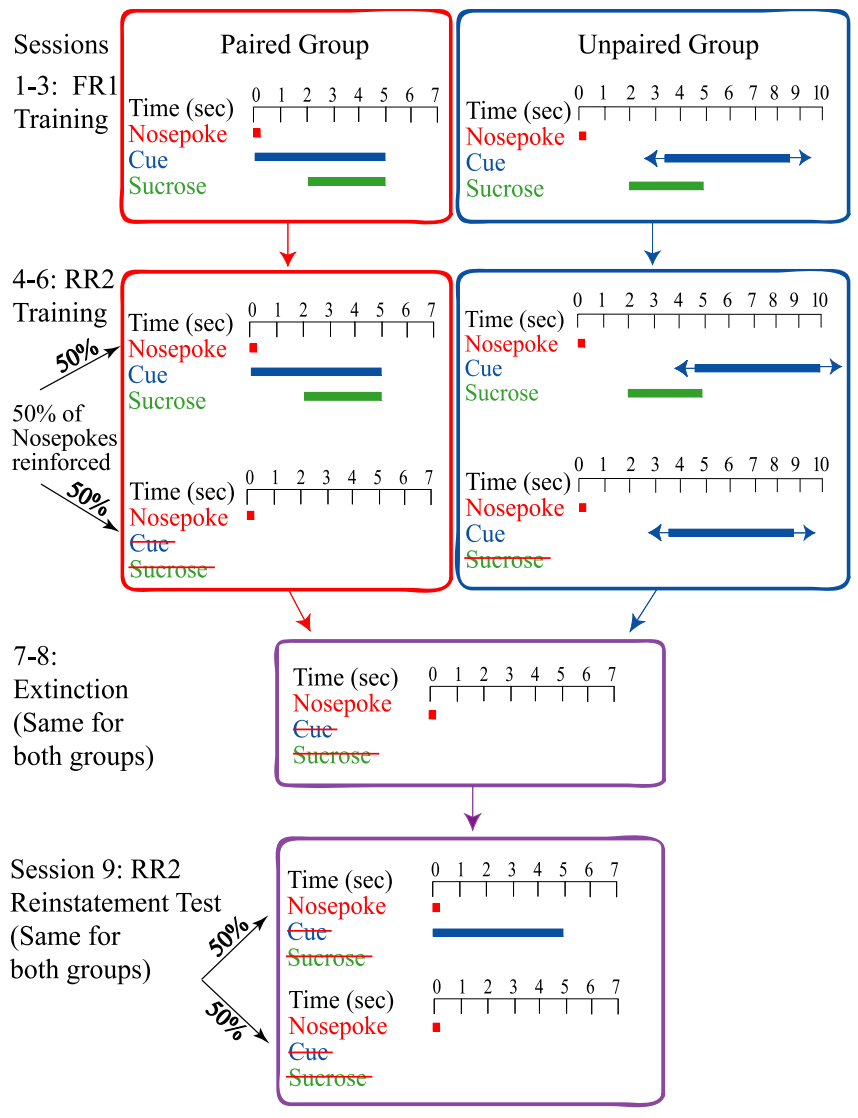

Figure 1. Diagram of behavioral paradigm. A cue-induced reinstatement paradigm was modified to allow dissociation of sensory and motor confounds. For the paired group, sucrose delivery was always paired with the LT-cue; for the unpaired group, the LT-cue was presented randomly. However, note that the LT-cue followed $50 \%$ of responses for both groups during the reinstatement session. In an FR1 schedule, every response is reinforced. In a random ratio 2 (RR2) schedule, each response has a 0.5 probability of being reinforced.

\section{Reward-associated cues are encoded in the BLA of animals that used the cue to guide behavior}

We recorded from a total of 212 BLA neurons during the reinstatement session, (paired group, 100 neurons; unpaired group, 112 neurons). Although there was no significant difference between paired and unpaired groups in the proportion of neurons responsive to port entries, there was a significant difference between groups in the proportion of neurons that responded to the cue. Twenty-seven neurons $(27 \%)$ recorded from the paired group showed a significant $(p<0.01)$ change in phasic activity selective for the cue; an example of such a neuron is shown in Figure 4. In contrast, the unpaired group had only seven such neurons $(6 \%)$, which was significantly different from the paired group ( 27 vs $\left.6 \% ; \chi^{2}=15.99 ; p<0.001\right)$. An additional population $(19 \% ; n=19)$ of neurons recorded from paired animals responded differentially to the cue presentation itself and to the interval during which time the cue was anticipated but was not presented after nosepoke responses with no cue; an example of such a neuron is shown in Figure $5 B$. Only one such neuron was identified in the unpaired group $(0.9 \%)$, which was significantly different from the paired group (19 vs $0.9 \% ; \chi^{2}=19.52 ; p<$ 0.001 ). Additionally, the mean percentages of neurons in both populations per rat were significantly different between groups (Fig. 6). Because rats in the paired group were more likely to go to the port after cue presentation (Fig. 3C), BLA neurons with differential responses to the presence or absence of the cue could contribute to the guidance of this behavior. 
Recordings made during training sessions confirm the presence of these cue responses during sessions in which the cue was paired with the reward. During the final training session, paired animals showed similar proportions of neurons that were selectively cue responsive (24\%) as well as differentially responsive to the presence and absence of the cue (16\%) as during reinstatement (Table 1).

\section{Differential encoding of the cue as an incentive motivator and conditioned reinforcer}

At the beginning of the reinstatement session, paired animals demonstrated a high rate of nosepoke responding (Fig. 3B), followed by a steady but protracted rate of responding as the session continued. In addition, port entries immediately after the cue tended to occur early in the session but not later in the reinstatement session (Fig. 5A). Notably, paired animals continued to respond to the cue even after they ceased checking the port for sucrose (Fig. $5 A$ ), suggesting that the cue has dual effects on behavior, acting both as a trigger for reward-directed behavior in early reinstatement and as a conditioned reinforcer throughout reinstatement. We used the change in latency between nosepoke and subsequent port entry as a criterion to distinguish between early and late reinstatement (for details, see Materials and Methods). Also noteworthy was the step-wise manner in which animals ceased postcue port checking (Fig. 5A) that occurred simultaneously with the discrete slope change of the rate of nosepoke responding between early and late reinstatement. The mean nosepoke response rate of paired animals was significantly greater during early reinstatement (mean nosepokes per $\min , 3.6 \pm 0.15$ ) than in late reinstatement (mean nosepokes per min, $0.31 \pm 0.06$; paired $t$ test; $p<0.001$ ), an example of which is shown in Figure $3 B$.

Interestingly, the majority of neurons that showed a differential response to the presence and absence of the cue typically lost their response to both the presence ( $n=18$ of 19 neurons; $94.7 \%)$ and the absence ( $n=17$ of 19 neurons; $89.5 \%$ ) of the cue after the animal ceased port checking in the reinstatement session (Fig. $5 B, C$ ).

Additional analysis revealed that a population of cueresponsive neurons $(34 \% ; n=34)$ in the paired group responded to cues followed by port entry but not to cues without subsequent port entry (Figs. $7 A, 8$ ). To determine whether this change was related to the motor activity of approaching the port, we looked at the neural response in the unpaired group. Because the cue did not guide the behavior of the unpaired animals, we included all trials, independent of cue presentation. Only three neurons $(2.7 \%)$ in the unpaired group showed a difference in activity after the nosepoke that depends upon whether the subject enters the port or not. Therefore, these responses from the paired subjects are unlikely to reflect movement to the port per se. Hence, this
B

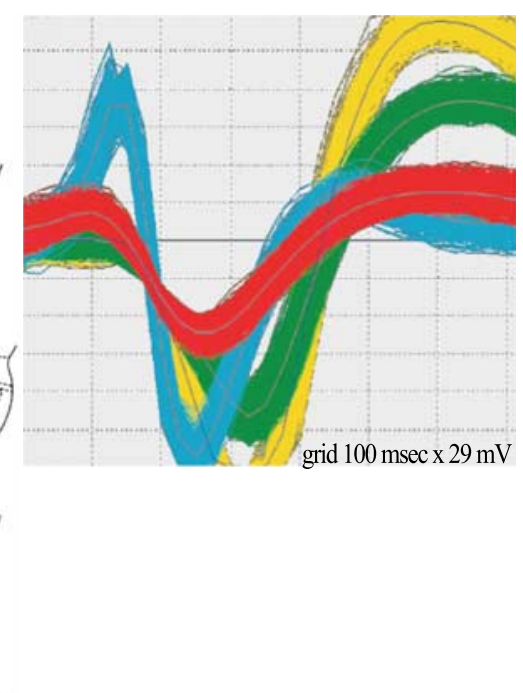

for the paire to bregma (Paxinos and Watson, 1998). $\boldsymbol{B}$, Example waveforms of single units recorded from a single electrode tip located in the BLA and isolated using principle component cluster analysis.

group of neurons in the paired subjects responded to the cue when the cue triggered the conditioned approach to the sucrose delivery port. Once the cue ceased to act as an incentive to approach the sucrose port, this population of neurons no longer showed a phasic change in activity, therefore indicating that these neurons encode conditioned incentive.

The remaining cue-responsive neurons $(n=12 ; 12 \%)$ recorded from the paired group maintained their cue response throughout the reinstatement session (Figs. 7B,8). The responsecontingent presentation of the cue acted as a conditioned reinforcer throughout the reinstatement session, as demonstrated by its ability to maintain continued responding in the absence of the primary reward. In contrast, in the unpaired group, the cue did not act as a conditioned incentive or as a conditioned reinforcer, as demonstrated by the low level of responding in the first $80 \mathrm{~min}$ of the session.

\section{Neurons exhibit increased bursting during reinstatement}

For analysis of burst characteristics, $1000 \mathrm{~s}$ intervals containing comparable levels of operant responding and cue presentations 


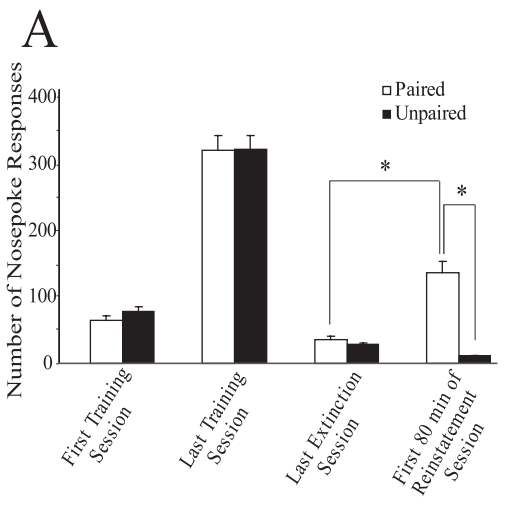

$\mathrm{C}$

B
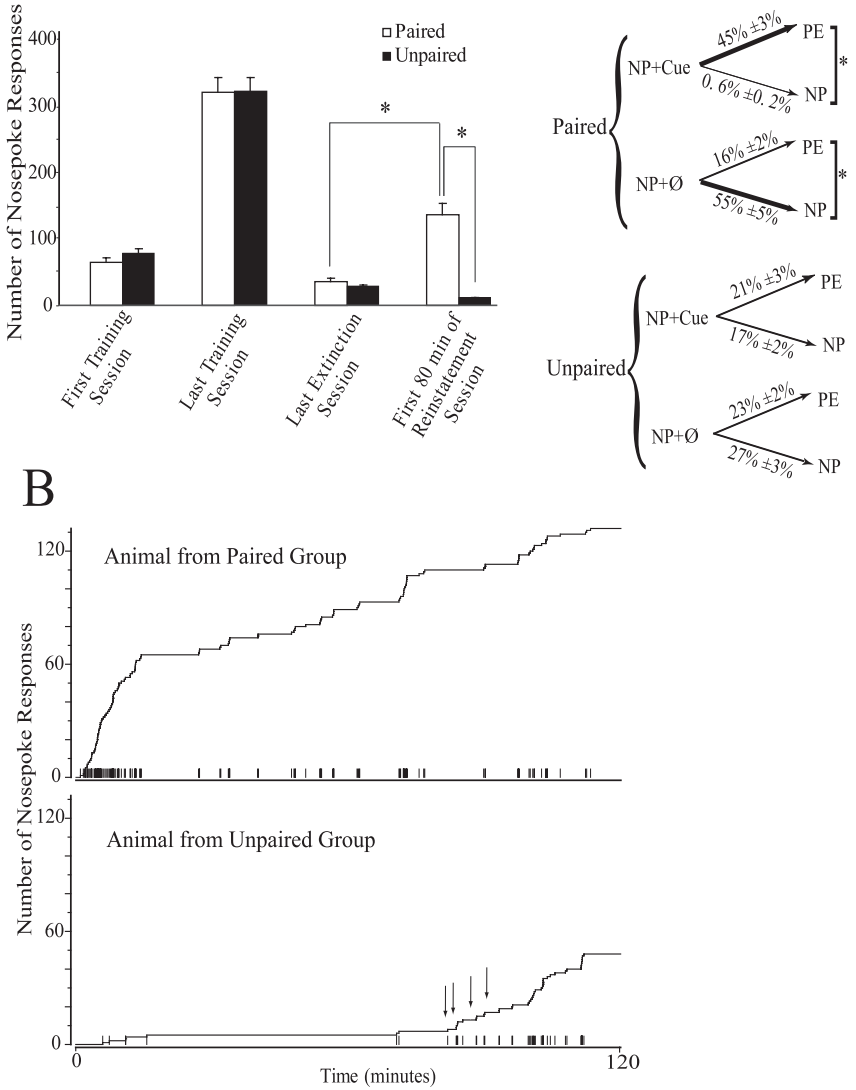

Figure 3. Behavioral differences between paired and unpaired groups. $A$, Mean numbers of responses ( \pm SEM) for paired $(n=5)$ and unpaired $(n=5)$ groups were not different during training sessions and extinction, but only paired animals showed a cue-induced reinstatement of responding. Data analyzed with ANOVA revealed the main effects of group $\left(F_{(1,16)}=16.23\right.$; $p<0.001)$, session $\left(F_{(1,16)}=6.57 ; p<0.03\right)$, and a group $\times$ session interaction $\left(F_{(1,16)}=\right.$ 13.36; $p<0.003$ ) and were followed by pairwise comparisons (Holm-Sidak). ${ }^{*} p<0.001 . B$, Cumulative activity records depicting the response pattern for representative animals of each group during the 120 min reinstatement session. Each upward inflection and corresponding tick mark represents one nosepoke response, shown on the $y$-axis. Note slope change for the paired animal after the first $\sim 65$ nosepoke responses. Arrows represent noncontingent sucrose deliveries for the unpaired group $80 \mathrm{~min}$ into the reinstatement session. C, Microanalysis of behavior during the reinstatement session. Mean ( \pm SEM) percentages of port entries or nosepokes are given. In the $2 \mathrm{~s}$ after the nosepoke, paired animals were more likely to enter the port after a cue (paired $t$ test; $p<0.005)$ and to repeat responding after the absence of a cue $(p<0.003)$, whereas unpaired animals were equally likely to enter the port or to repeat nosepoking after either the presence or absence of the cue ( $p>0.05)$. NP, nosepoke; $\emptyset$, absence of cue; PE, port entry. ${ }^{*} p<0.005$.

were selected from the reinstatement session for each group. We found that the paired group showed a significantly higher number of bursts, bursts per second, and percentage of spikes found in bursts relative to the unpaired group (Table 2). However, there were no significant differences between groups in overall mean firing rate, burst duration, or mean number of spikes found in a burst (Table 2).

\section{Electrophysiological characterization of neuronal response types}

Pyramidal projection neurons compose $\sim 95 \%$ of the neurons in the BLA, the remainder being interneurons (McDonald 1982, 1984). Studies have shown that pyramidal neurons and interneurons can be differentiated by both morphological and electrophysiological characteristics (Washburn and Moises, 1992; Rain-

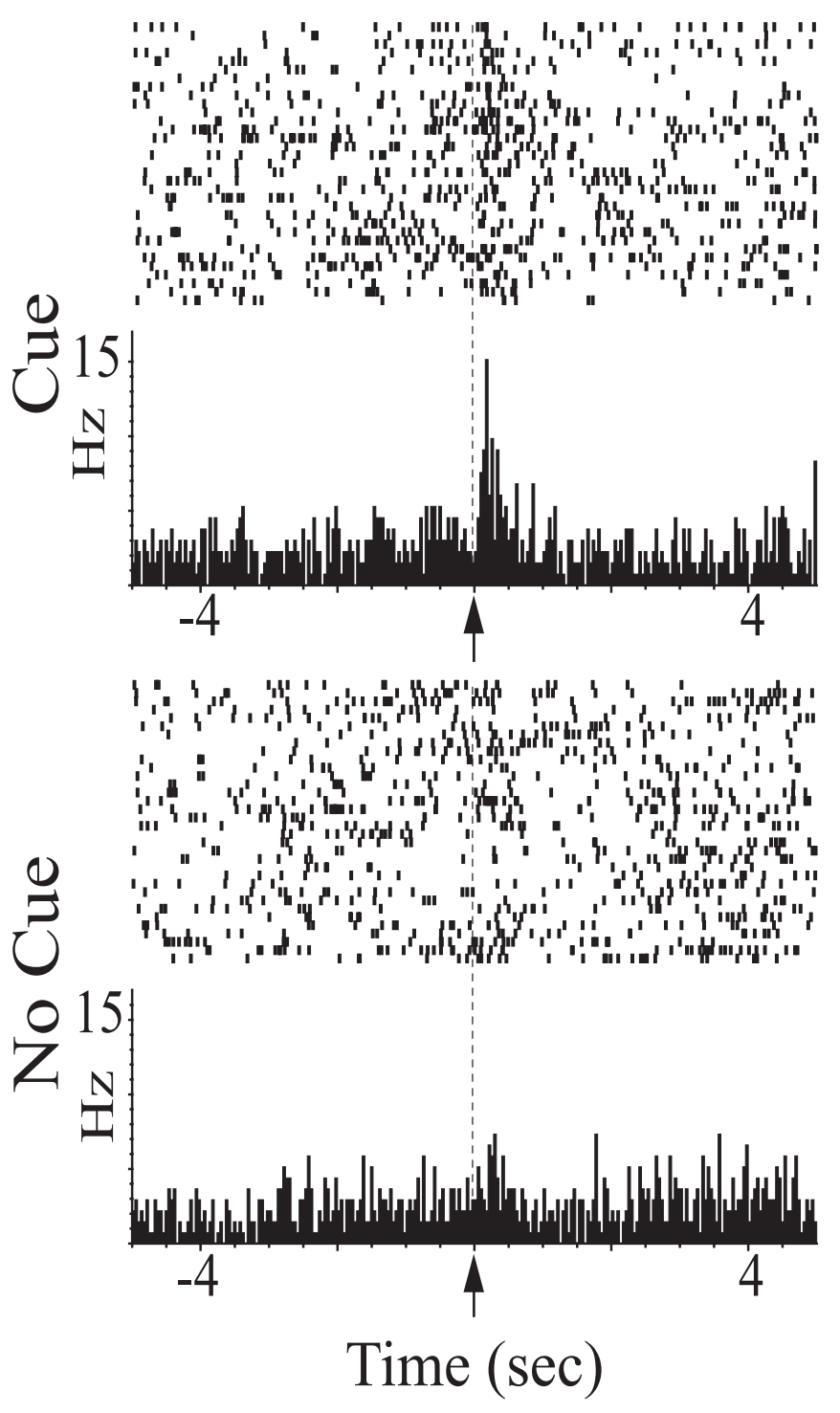

Figure 4. Example of a selectively cue-responsive neuron. The perievent raster and histogram (PERH) of a neuron showed a significant change in phasic activity in response to nosepokes paired with a cue presentation but did not show a significant change in phasic activity to nosepokes that were not paired with the cue. Neurons showing this response pattern are referred to as selectively cue responsive. For all PERHs, the arrow indicates the time of the reference event, or zero time point. The bin size equals $40 \mathrm{~ms}$ for this and subsequent figures.

nie et al., 1993). These studies have shown that pyramidal neurons have longer-duration action potentials, very low spontaneous firing rates, and fewer spikes per burst. Interneurons have shorter-duration action potentials, very high spontaneous firing rates, and no sign of accommodation (Washburn and Moises, 1992). Using these electrophysiological characteristics, we identified six putative interneurons of 100 neurons from the paired group. None of these putative interneurons were phasically responsive to cue presentations or nosepokes.

\section{Discussion}

We report that neurons in the BLA encode a cue that induces relapse to reward-seeking behavior, and we posit that these cue responses in the BLA encode two distinct behavioral functions of conditioned cues: incentive motivation and conditioned reinforcement. Neurons that responded differentially to the presence and absence of the cue were likely to contribute to the guidance of 


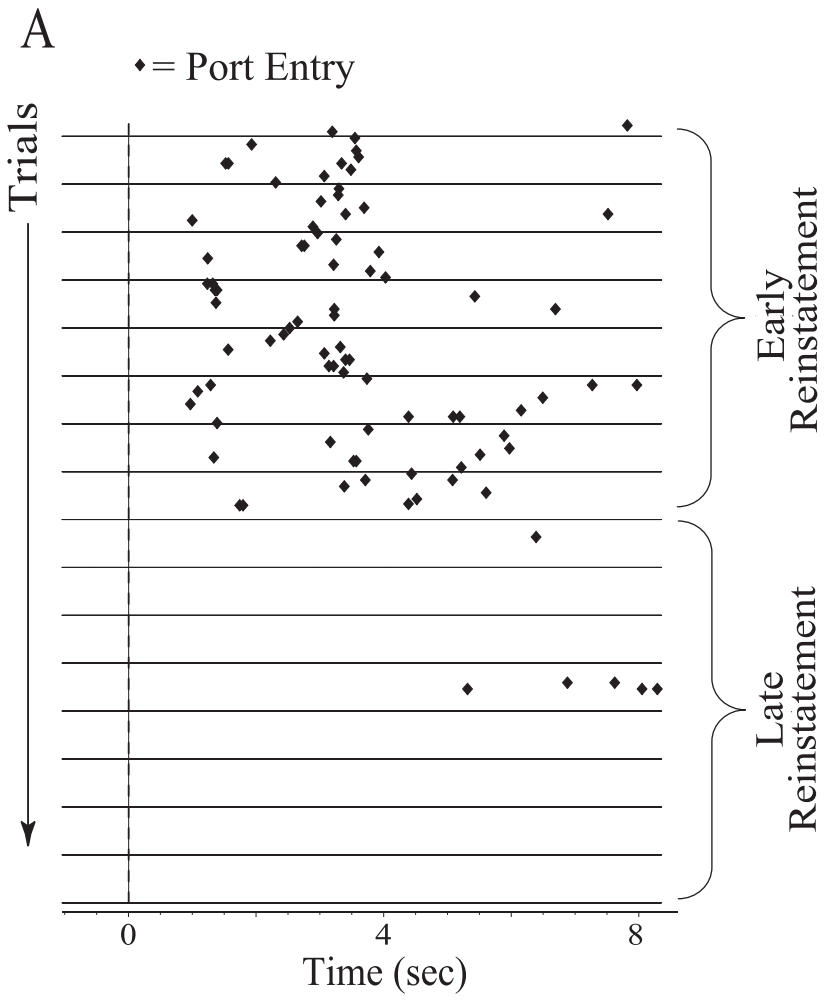

B

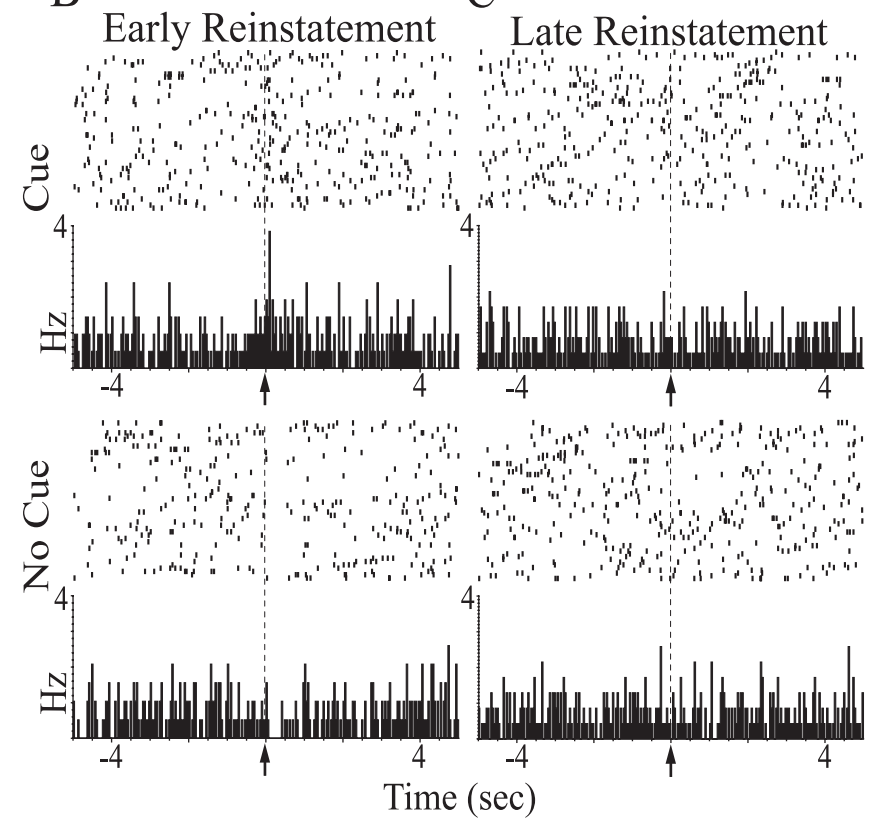

Figure 5. Corresponding changes in behavior and neural encoding of the cue from early to late reinstatement. $A$, Example of the pattern of port entry during the reinstatement session for a representative paired animal shown in a perievent raster with response-contingent cue presentation as the reference event. Each row represents a trial, and the cue presentation is the reference event. Early in the session, cue presentations were consistently followed by port entries (filled diamonds). A discrete change occurred in late reinstatement because the paired animal continued to respond to the cue but no longer checked the port for sucrose. $\boldsymbol{B}, \boldsymbol{C}$, Perievent raster and histogram (PERH) of a BLA single-unit with a differential response to the presence (excitation) and absence (inhibition) of the cue in early reinstatement $(\boldsymbol{B})$ and then a loss of these responses in late reinstatement (C).

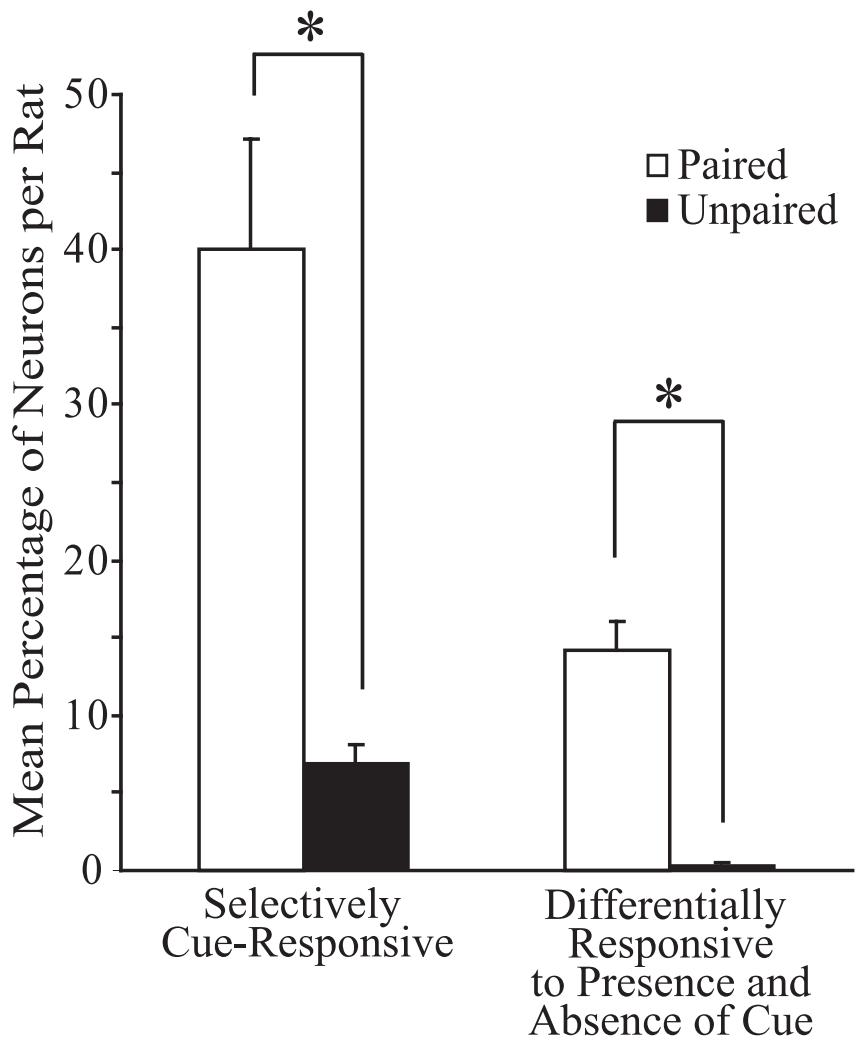

Figure 6. Neural responses to cue presentation. Two types of cue-responsive neurons include selectively cue-responsive neurons and neurons that were differentially responsive to the presence and absence of the cue. The paired group had significantly higher mean percentages of neurons per rat selectively responding to the cue, as well as mean percentages of neurons differentially responding to the presence or absence of the cue, than the unpaired group ( $n=$ 5 rats per group; ${ }^{*} p<0.001 ; t$ test).

Table 1. Neural responses from the paired group during the final training session indicate similar proportions of cue-responsive neurons as during reinstatement

\begin{tabular}{llc}
\hline & \multicolumn{2}{l}{ Session } \\
\cline { 2 - 3 } & Training & Reinstatement \\
\hline Total neurons recorded & 88 & 100 \\
Cue responsive & $35(40 \%)$ & $46(46 \%)$ \\
Selectively cue responsive & $21(24 \%)$ & $27(27 \%)$ \\
Differentially cue responsive & $14(16 \%)$ & $19(19 \%)$ \\
\hline
\end{tabular}

subsequent behavioral choices and primarily overlapped with incentive motivation-encoding neurons.

We termed the rapidly extinguishing population of neurons "incentive motivation encoding" because the activation of these neurons by the cue triggered a motivated behavioral state during which nosepoke responses and subsequent port entries were performed at a high rate. The slowly extinguishing population of neurons that responded to the cue throughout reinstatement was termed "conditioned reinforcement encoding," because the activation of these neurons continued as long as repeated nosepoking was performed, even after the incentive-triggered port-checking behavior was extinguished.

Our behavioral task gave rats the opportunity to demonstrate whether they were working for sucrose or only cue presentations. Multiple behavioral events were measured, including not only the instrumental response (nosepoke) but also a measure of conditioned approach (port entry). This allowed the distinction between trials in which the cue acted as a conditioned incentive, 


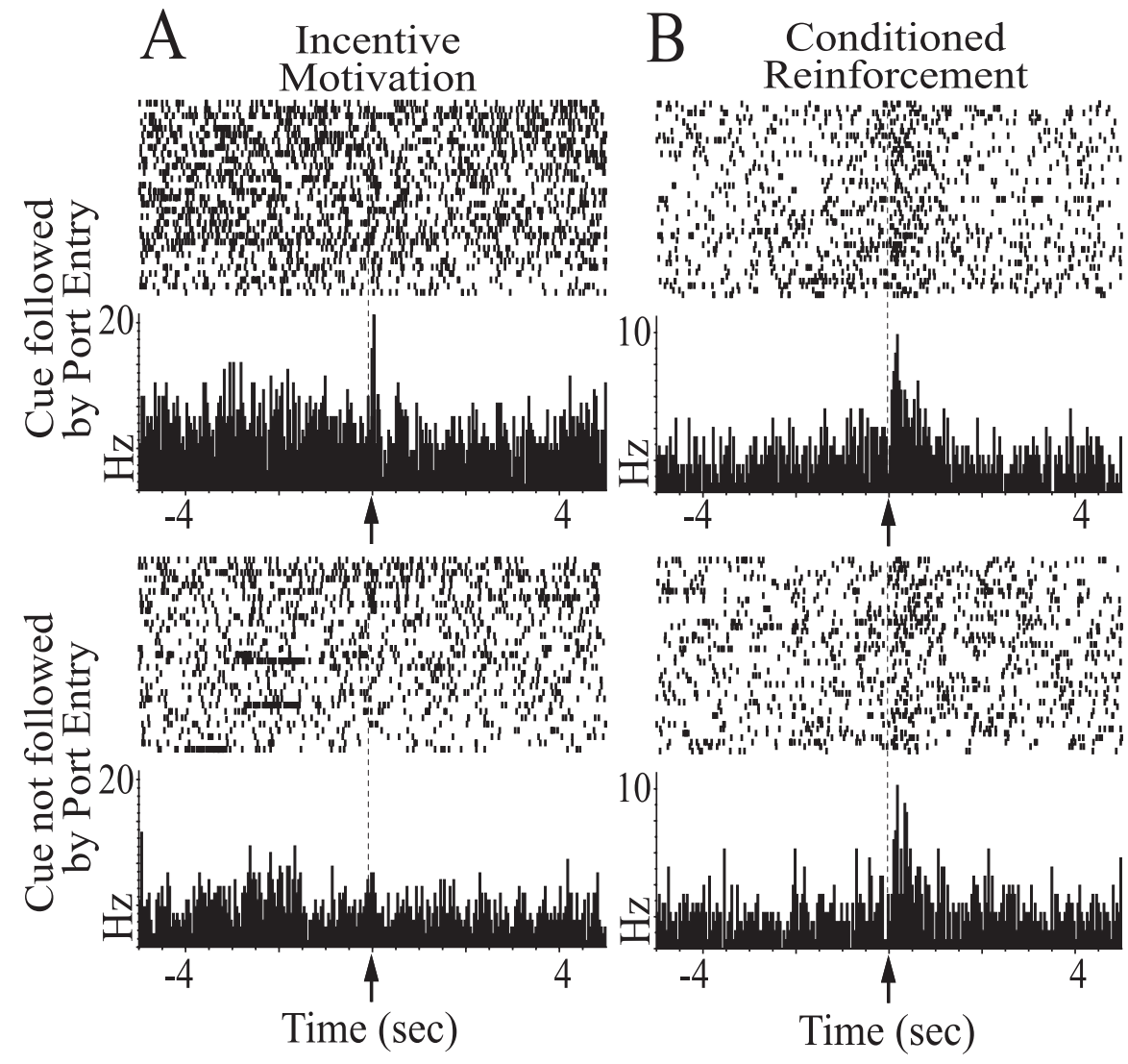

Figure 7. Cue-responsive neurons can be categorized into rapidly extinguishing or long-lasting response types. $A$, Perievent raster and histogram (PERH) of BLA single-unit encoding conditioned incentive; the phasic response to the cue is present only when the subject entered the port after cue presentation. Once the cue no longer provided an incentive to approach the sucrose port, the response to the cue disappeared. $B$, PERH of BLA single-unit encoding conditioned reinforcement; the phasic response to the cue is present whether or not the subject entered the port after cue presentation. The cue acted as a conditioned reinforcer throughout the reinstatement session, as shown by the ability of the cue presentation to maintain a steady rate of nosepoke responding in the absence of the primary reinforcer.

$\square$ Incentive Motivation Encoding $\square$ Conditioned Reinforcement Encoding

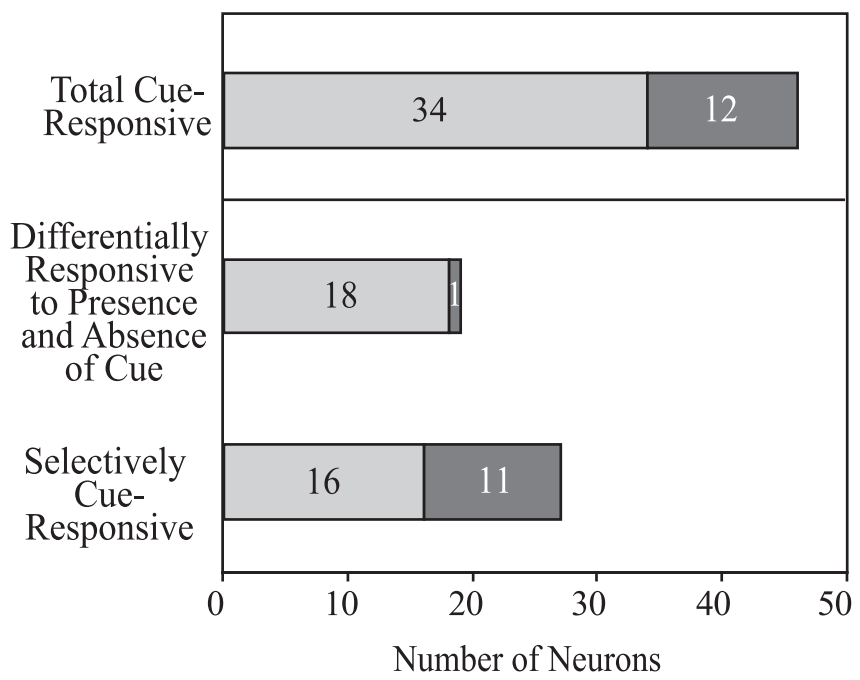

Figure 8. Subpopulations of cue-responsive neurons. The distribution of numbers of cueresponsive neurons recorded from animals in the paired group that are conditioned incentive encoding or conditioned reinforcement encoding. triggering a motivated reward-seeking state, and trials in which the cue acted as a conditioned reinforcer, supporting continued nosepoke responding. Hence, it was possible to track single units as different acquired properties of the cue guided the behavior of animals in the paired group within a single session.

At the beginning of the reinstatement session, the paired animals displayed a high rate of responding, followed by a discrete rate change (Fig. $3 B$ ) that temporally corresponds with the step-wise cessation of postcue port checking (Fig. $5 A, B$ ). In temporal agreement with this sudden behavioral change, a subpopulation of neurons ceases responding to the cue. We speculate that this change in the rate of responding occurs because initially both the incentive and reinforcement encoding populations of neurons are supporting the nosepoking behavior, and later, when the incentive-encoding population of neurons stops responding to the cue, the response rate changes, perhaps reflecting that only one population of neurons is now supporting nosepoke responding. Our hypothesis that BLA neurons are guiding reward-seeking behavior is supported by the coincident decrease in the behavioral response rate and the proportion of cue-responsive neurons.

Both the incentive motivationencoding and conditioned reinforcementencoding populations of neurons responded to the cue while the cue-reward association was still intact, but as the meaning of the cue became ambiguous, as demonstrated by the cessation of postcue port checking (Fig. $5 A$ ), one population of cue-responsive neurons ceased responding to the cue, which suggests that these neurons are encoding incentive motivation, as measured by cue-triggered approach and cued relapse. Lesion studies show that the BLA is important for outcome-specific incentive processes (Balleine et al., 2003; Corbit and Balleine, 2005). Interestingly, most of the conditioned incentive-encoding neurons were differentially responsive to both the presence and absence of the cue. Once the animal's behavior was no longer guided by the presence or absence of the cue (when the animal ceased port checking) this population of neurons no longer displayed the differential change in phasic activity. In agreement with a role for the BLA in conditioned reinforcement (Cador et al., 1989), the second population of cueresponsive neurons maintained their cue response while instrumental responding continued, perhaps reflecting the conditioned-reinforcing properties of the reward-paired cue that can motivate continued nosepoking even after the animals have stopped checking the reward port.

The current findings confirm that the BLA is a site for the neural representation of reward-associated cues that induce reinstatement to reward-seeking behavior, consistent with studies showing that the BLA is critically involved in cue-induced reinstatement (Meil and See, 1997; Fuchs and See, 2002; Yun and Fields, 2003). Importantly, it has been shown that varying with- 
drawal periods can affect extinction behavior and the degree to which cueinduced reinstatement is expressed (Grimm et al., 2002; Myers et al., 2006). Although our results represent the first evidence that distinct populations of neurons encode incentive and reinforcing properties of a CS, additional studies are needed to dissect relevant issues. Our findings provide an impetus for additional experimentation regarding BLA neural activity during distinct behavioral tests of incentive motivation and conditioned reinforcement and the persistence of these neural responses with varying withdrawal periods.

The finding that two populations of cue-responsive BLA neurons are active during reinstatement (Fig. 8) is reminiscent of studies done with extinction of cue-shock associations (Repa et al., 2001). In the extinction of a CS-shock association, it was shown that there were two subpopulations of neurons in the lateral amygdala with CS-evoked firing rate changes, one population that exhibited increased responses to the CS during early extinction and then fell back to baseline levels in late extinction and one that maintained their increased responses to the CS throughout extinction (Repa et al., 2001). In our paradigm, the reinstatement session was a session in which the cue-reward association was extinguished, similar to the extinction session from the paradigm of Repa et al. (2001). Our findings are consistent with the Repa et al. (2001) study, showing analogous characteristics for two populations of BLA neurons that display either transient or long-lasting responses to the CS. This suggests that BLA neurons may encode appetitive and aversive stimuli similarly. Because there is evidence that distinct populations of BLA neurons encode appetitive and aversive stimuli (Paton et al., 2006), it is likely that these neurons have different projections and that the function of the BLA lies in the absolute valence of the outcomes predicted by sensory stimuli, and positive or negative value is processed elsewhere.

The present findings are also consistent with findings from Carelli et al. (2003) who recorded in the BLA during selfadministration of cocaine. They described three typical response types, including responses that occurred during cue presentation. The present study extends this report by examining the response of BLA neurons to the reward-associated cue during reinstatement in the absence of the primary reinforcer and by dissociating the motor and sensory responses from the neural encoding of the cue-reward association.

The BLA works in conjunction with downstream areas in the reward circuit by supplying them with information about the motivating and/or reinforcing properties of the cue to decide upon, plan, and execute appropriate behavioral outputs. For example, the BLA has robust reciprocal projections with the medial prefrontal cortex (mPFC) (Vertes, 2004). The mPFC is also important for cue-induced reinstatement of reward-seeking behavior (McLaughlin and See, 2003). Evidence suggests that the $\mathrm{mPFC}$ regulates the responsiveness of BLA neurons to odor cues previously associated with footshock (Rosenkranz et al., 2003). Additionally, the BLA transmits information about footshockconditioned stimuli to a subpopulation of mPFC neurons that encodes information about these emotionally salient cues with increased bursting activity (Laviolette et al., 2005). Our data showing a significant increase in the number of bursts, bursts per second, and percentage of total spikes that occur in bursts in the
BLA neurons of the paired group as compared with the unpaired group (Table 2) suggest that the reward-paired cue may induce bursting of BLA neurons and are consistent with the study done by Laviolette et al. (2005). These results suggest that the cueinduced bursts seen in the MPFC may originate in the BLA.

The orbitofrontal cortex (OFC), which is reciprocally connected to the BLA (Kita and Kitai, 1990; Shi and Cassell, 1998), is thought to work in coordination with the BLA to use incentive information to guide behavior (Gallagher and Schoenbaum, 1999; Schoenbaum et al., 1999, 2003; Baxter et al., 2000). It has been shown that lesions of either the BLA or OFC alter the neural encoding of information about expected outcomes and acquired value (Schoenbaum et al., 2003; Saddoris et al., 2005). In addition, the BLA sends inputs to the nucleus accumbens core (Kelley et al., 1982; Wright et al., 1996), and it has been reported that these connections are important for performing second-order conditioned responses (Setlow et al., 2002). The nucleus accumbens has been suggested as a "final common pathway" for all types of reinstatement (cue-induced, drug-induced, stressinduced) (Kalivas and McFarland, 2003). For these reasons, understanding how the BLA encodes cues that guide goal-directed behavior is crucial to understanding how our brains process sensory stimuli that induce relapse to reward-seeking behavior.

\section{Conclusion}

In conclusion, our data demonstrate that BLA neurons show changes in phasic activity to the response-contingent presentation of a cue that induces reinstatement of reward-seeking behavior. BLA neurons also respond to the absence of the anticipated cue. Importantly, distinct neuronal populations within the BLA encode incentive motivation and conditioned reinforcement.

The activity of these neural populations can motivate reward seeking in the absence of reward, and serve to guide goal-oriented behaviors. These findings represent significant progress toward understanding the neural circuitry encoding cues that can reinstate extinguished reward-seeking behavior. This increased understanding will aid in the development of therapeutic interventions for conditioned-cued relapse of reward-seeking behaviors in humans, including compulsive eating disorders and drug addiction.

\section{References}

Arroyo M, Markou A, Robbins TW, Everitt BJ (1998) Acquisition, maintenance and reinstatement of intravenous cocaine self-administration under a second-order schedule of reinforcement in rats: effects of conditioned cues and continuous access to cocaine. Psychopharmacology (Berl) 140:331-344.

Balleine BW, Killcross AS, Dickinson A (2003) The effect of lesions of the basolateral amygdala on instrumental conditioning. J Neurosci 23:666-675.

Baxter MG, Parker A, Lindner CC, Izquierdo AD, Murray EA (2000) Control of response selection by reinforcer value requires interaction of amygdala and orbital prefrontal cortex. J Neurosci 20:4311-4319. 
Berridge KC, Robinson TE (2003) Parsing reward. Trends Neurosci 26:507-513.

Cador M, Robbins TW, Everitt BJ (1989) Involvement of the amygdala in stimulus-reward associations: interaction with the ventral striatum. Neuroscience 30:77-86.

Carelli RM, Williams JG, Hollander JA (2003) Basolateral amygdala neurons encode cocaine self-administration and cocaine-associated cues. J Neurosci 23:8204-8211.

Childress A, Ehrman R, McLellan AT, O’Brien C (1988) Conditioned craving and arousal in cocaine addiction: a preliminary report. NIDA Res Monogr 81:74-80.

Childress AR, Mozley PD, McElgin W, Fitzgerald J, Reivich M, O’Brien CP (1999) Limbic activation during cue-induced cocaine craving. Am J Psychiatry 156:11-18.

Corbit LH, Balleine BW (2005) Double dissociation of basolateral and central amygdala lesions on the general and outcome-specific forms of pavlovian-instrumental transfer. J Neurosci 25:962-970.

Davis M (1992) The role of the amygdala in conditioned fear. In: The amygdala: neurological aspects of emotion, memory, and mental dysfunction (Aggleton JP, ed), pp 255-306. Chichester, UK: Wiley.

Davis WM, Smith SG (1976) Role of conditioned reinforcers in the initiation, maintenance and extinction of drug-seeking behavior. Pavlov J Biol Sci 11:222-236.

Ehrman RN, Robbins SJ, Childress AR, O’Brien CP (1992) Conditioned responses to cocaine-related stimuli in cocaine abuse patients. Psychopharmacology (Berl) 107:523-529.

Epstein DH, Preston KL, Stewart J, Shaham Y (2006) Toward a model of drug relapse: an assessment of the validity of the reinstatement procedure. Psychopharmacology (Berl).

Everitt BJ, Robbins TW (2000) Second-order schedules of drug reinforcement in rats and monkeys: measurement of reinforcing efficacy and drugseeking behaviour. Psychopharmacology (Berl) 153:17-30.

Everitt BJ, Robbins TW (2005) Neural systems of reinforcement for drug addiction: from actions to habits to compulsion. Nat Neurosci 8:1481-1489.

Everitt BJ, Cador M, Robbins TW (1989) Interactions between the amygdala and ventral striatum in stimulus-reward associations: studies using a second-order schedule of sexual reinforcement. Neuroscience 30:63-75.

Fuchs RA, See RE (2002) Basolateral amygdala inactivation abolishes conditioned stimulus- and heroin-induced reinstatement of extinguished heroin-seeking behavior in rats. Psychopharmacology (Berl) 160:425-433.

Gallagher M (2000) The amygdala and associative learning. In: The amygdala: a functional analysis (Aggleton JP, ed), pp 311-323. New York: Oxford UP.

Gallagher M, Schoenbaum G (1999) Functions of the amygdala and related forebrain areas in attention and cognition. Ann $N$ Y Acad Sci 877:397-411.

Grimm JW, Shaham Y, Hope BT (2002) Effect of cocaine and sucrose withdrawal period on extinction behavior, cue-induced reinstatement, and protein levels of the dopamine transporter and tyrosine hydroxylase in limbic and cortical areas in rats. Behav Pharmacol 13:379-388.

Kalivas PW, McFarland K (2003) Brain circuitry and the reinstatement of cocaine-seeking behavior. Psychopharmacology (Berl) 168:44-56.

Kelley AE, Domesick VB, Nauta WJ (1982) The amygdalostriatal projection in the rat-an anatomical study by anterograde and retrograde tracing methods. Neuroscience 7:615-630.

Kita H, Kitai ST (1990) Amygdaloid projections to the frontal cortex and the striatum in the rat. J Comp Neurol 298:40-49.

Laviolette SR, Lipski WJ, Grace AA (2005) A subpopulation of neurons in the medial prefrontal cortex encodes emotional learning with burst and frequency codes through a dopamine D4 receptor-dependent basolateral amygdala input. J Neurosci 25:6066-6075.

LeDoux J (1996) Emotional networks and motor control: a fearful view. Prog Brain Res 107:437-446.

McDonald AJ (1982) Neurons of the lateral and basolateral amygdaloid nuclei: a Golgi study in the rat. J Comp Neurol 212:293-312.
McDonald AJ (1984) Neuronal organization of the lateral and basolateral amygdaloid nuclei in the rat. J Comp Neurol 222:589-606.

McGaugh JL (2002) Memory consolidation and the amygdala: a systems perspective. Trends Neurosci 25:456.

McLaughlin J, See RE (2003) Selective inactivation of the dorsomedial prefrontal cortex and the basolateral amygdala attenuates conditioned-cued reinstatement of extinguished cocaine-seeking behavior in rats. Psychopharmacology (Berl) 168:57-65.

Meil WM, See RE (1997) Lesions of the basolateral amygdala abolish the ability of drug associated cues to reinstate responding during withdrawal from self-administered cocaine. Behav Brain Res 87:139-148.

Myers KM, Ressler KJ, Davis M (2006) Different mechanisms of fear extinction dependent on length of time since fear acquisition. Learn Mem 13:216-223.

Nie H, Janak PH (2003) Comparison of reinstatement of ethanol- and sucrose-seeking by conditioned stimuli and priming injections of allopregnanolone after extinction in rats. Psychopharmacology (Berl) 168:222-228.

O’Brien CP, Childress AR, Ehrman R, Robbins SJ (1998) Conditioning factors in drug abuse: can they explain compulsion? J Psychopharmacol 12:15-22.

Paton JJ, Belova MA, Morrison SE, Salzman CD (2006) The primate amygdala represents the positive and negative value of visual stimuli during learning. Nature 439:865-870.

Paxinos G, Watson C (1998) The rat brain in stereotaxic coordinates. New York: Academic.

Quirk GJ, Repa C, LeDoux JE (1995) Fear conditioning enhances shortlatency auditory responses of lateral amygdala neurons: parallel recordings in the freely behaving rat. Neuron 15:1029-1039.

Rainnie DG, Asprodini EK, Shinnick-Gallagher P (1993) Intracellular recordings from morphologically identified neurons of the basolateral amygdala. J Neurophysiol 69:1350-1362.

Repa JC, Muller J, Apergis J, Desrochers TM, Zhou Y, LeDoux JE (2001) Two different lateral amygdala cell populations contribute to the initiation and storage of memory. Nat Neurosci 4:724-731.

Rosenkranz JA, Moore H, Grace AA (2003) The prefrontal cortex regulates lateral amygdala neuronal plasticity and responses to previously conditioned stimuli. J Neurosci 23:11054-11064.

Saddoris MP, Gallagher M, Schoenbaum G (2005) Rapid associative encoding in basolateral amygdala depends on connections with orbitofrontal cortex. Neuron 46:321-331.

Schoenbaum G, Chiba AA, Gallagher M (1999) Neural encoding in orbitofrontal cortex and basolateral amygdala during olfactory discrimination learning. J Neurosci 19:1876-1884.

Schoenbaum G, Setlow B, Saddoris MP, Gallagher M (2003) Encoding predicted outcome and acquired value in orbitofrontal cortex during cue sampling depends upon input from basolateral amygdala. Neuron 39:855-867.

Setlow B, Holland PC, Gallagher M (2002) Disconnection of the basolateral amygdala complex and nucleus accumbens impairs appetitive pavlovian second-order conditioned responses. Behav Neurosci 116:267-275.

Shi CJ, Cassell MD (1998) Cortical, thalamic, and amygdaloid connections of the anterior and posterior insular cortices. J Comp Neurol 399:440-468.

Uwano T, Nishijo H, Ono T, Tamura R (1995) Neuronal responsiveness to various sensory stimuli, and associative learning in the rat amygdala. Neuroscience 68:339-361.

Vertes RP (2004) Differential projections of the infralimbic and prelimbic cortex in the rat. Synapse 51:32-58.

Washburn MS, Moises HC (1992) Electrophysiological and morphological properties of rat basolateral amygdaloid neurons in vitro. J Neurosci 12:4066-4079.

Wright CI, Beijer AV, Groenewegen HJ (1996) Basal amygdaloid complex afferents to the rat nucleus accumbens are compartmentally organized. J Neurosci 16:1877-1893.

Yun IA, Fields HL (2003) Basolateral amygdala lesions impair both cue- and cocaine-induced reinstatement in animals trained on a discriminative stimulus task. Neuroscience 121:747-757. 